\title{
Diagnostic blocks-a pain in the back?
}

The use of diagnostic blocks before radiofrequency denervation in patients with lumbar fractogenic pain is unnecessary, cost-ineffective and prolongs the treatment time for patients. These findings, from a study by Steven Cohen and colleagues, might have important clinical and economic implications for patients with low back pain.

The investigators randomly assigned 151 patients with low back pain to receive radiofrequency denervation in the lumbar $\mathrm{z}$-joint on the basis of clinical findings alone (group 0) or a positive response to one or two confirmatory diagnostic blocks (groups 1 and 2, respectively). Overall, the predesignated positive outcome of $\geq 50 \%$ pain relief lasting for 3 months was achieved by $33 \%$ of patients in group 0 , but only $16 \%$ and $22 \%$ of patients in groups 1 and 2, respectively. Success rates increased to $39 \%$ (group 1) and $64 \%$ (group 2) when only patients who proceeded to radiofrequency denervation were considered.
To assess the economical implications of including diagnostic blocks in the treatment of these patients, the researchers calculated the overall cost per successful treatment-approximately US\$6,286, US $\$ 17,142$ and US $\$ 15,241$ for groups 0,1 and 2 , respectively.

Cohen et al. conclude that although most relevant guidelines endorse the use of two confirmatory diagnostic blocks prior to radiofrequency denervation, bypassing diagnostic blocks is associated with the highest overall success rate and the lowest cost per successful procedure. However, the authors stress that diagnostic blocks should not be discontinued altogether as they are still useful for diagnosis before other spinal interventions.

\section{Rowan Higgs}

Original article Cohen, S. P. et al. Multicenter, randomized, comparative cost-effectiveness study comparing 0,1 , and 2 diagnostic medial branch (facet joint nerve) block treatment paradigms before lumbar facet radiofrequency denervation. Anesthesiology 113, 395-405 (2010) 